\title{
BMJ
}

\section{Contribution of smoking during pregnancy to inequalities in stillbirth and infant death in Scotland 1994-2003: retrospective population based study using hospital maternity records}

\author{
Ron Gray, clinical epidemiologist, ${ }^{1}$ Sandra R Bonellie, lecturer in statistics, ${ }^{2}$ James Chalmers, consultant in \\ public health medicine, ${ }^{3}$ lan Greer, dean, ${ }^{4}$ Stephen Jarvis, emeritus professor, ${ }^{5}$ Jennifer J Kurinczuk, reader in \\ perinatal epidemiology, ${ }^{1}$ Claire Williams, statistician ${ }^{2}$
}

\begin{abstract}
${ }^{1}$ National Perinatal Epidemiology Unit, University of Oxford, Oxford OX3 7LF

${ }^{2}$ School of Accountancy, Economics and Statistics, Napier University, Edinburgh EH10 5DT

${ }^{3}$ Information Services Division (ISD), NHS National Services Scotland, Edinburgh EH12 9EB

${ }^{4}$ Hull York Medical School, University of York, York Y010 5DD

${ }^{5}$ Sir lames Spence Institute of Child Health, Royal Victoria Infirmary, Newcastle upon Tyne NE1 4LP
\end{abstract}

Correspondence to: R Gray

ron.gray@npeu.ox.ac.uk

Cite this as: BMJ 2009:339:b3754 doi:10.1136/bmi.b3754

\section{ABSTRACT}

Objective To quantify the contribution of smoking during pregnancy to social inequalities in stillbirth and infant death. Design Population based retrospective cohort study. Setting Scottish hospitals between 1994 and 2003. Participants Records of 529317 singleton live births and 2699 stillbirths delivered at 24-44 weeks' gestation in Scotland from 1994 to 2003.

Main outcome measures Rates of stillbirth and infant, neonatal, and post-neonatal death for each deprivation category (fifths of postcode sector Carstairs-Morris scores); contribution of smoking during pregnancy ("no," "yes," or "not known") in explaining social inequalities in these outcomes.

Results The stillbirth rate increased from 3.8 per 1000 in the least deprived group to 5.9 per 1000 in the most deprived group. For infant deaths, the rate increased from 3.2 per 1000 in the least deprived group to 5.4 per 1000 in the most deprived group. Stillbirths were $56 \%$ more likely (odds ratio $1.56,95 \%$ confidence interval 1.38 to 1.77) and infant deaths were $72 \%$ more likely $(1.72,1.50$ to 1.97) in the most deprived compared with the least deprived category. Smoking during pregnancy accounted for $38 \%$ of the inequality in stillbirths and $31 \%$ of the inequality in infant deaths.

Conclusions Both tackling smoking during pregnancy and reducing infants' exposure to tobacco smoke in the postnatal environment may help to reduce stillbirths and infant deaths overall and to reduce the socioeconomic inequalities in stillbirths and infant deaths perhaps by as much as $30-40 \%$. However, action on smoking on its own is unlikely to be sufficient and other measures to improve the social circumstances, social support, and health of mothers and infants are needed.

\section{INTRODUCTION}

Both stillbirth and infant mortality show a social gradient within developed countries. ${ }^{1-6}$ Furthermore, the Department of Health in England uses the closing of the relative gap in infant mortality between the routine and manual occupational group and the population as a whole as a key public health target. ${ }^{7}$ As smoking during pregnancy has been clearly linked to stillbirths and infant deaths, ${ }^{8-11}$ and as smoking rates during pregnancy vary markedly with socioeconomic position, ${ }^{12}$ jointly exploring the effects of smoking and those of socioeconomic position is of interest. This will answer the question of how much of an effect smoking during pregnancy has on the social inequalities gap in stillbirths and infant deaths. Few studies have examined this question directly, ${ }^{13-15}$ but it is important in quantifying not only how many deaths might be avoided by intervening to prevent or reduce smoking during pregnancy but also whether the social gradient in stillbirths and infant deaths could be attenuated by antismoking measures. We have previously shown that smoking during pregnancy may have contributed to some of the social gradients in preterm birth in Scotland in the period 1994-2003. ${ }^{16}$ Here, we examine the effects of smoking on social gradients in stillbirths and infant deaths. Specifically, our aim was to study the effect of area deprivation on rates of stillbirth and infant mortality in Scotland for the 10 year period 1994-2003 and to establish whether smoking during pregnancy contributed to these gradients and, if so, to what extent.

\section{METHODS}

Information on all maternity admissions to all Scottish hospitals is recorded on Scottish morbidity record forms (SMR02), which are collated on a national database. This database contains information on demographic characteristics, including maternal place of residence clinical details of care, and data on birth outcomes such as gestational age at delivery. As less than $1 \%$ of births take place outside of hospital in Scotland, this database effectively allows the construction of a pregnancy and birth cohort study of all maternities in a general population of just over five million people containing around one million women of reproductive age and 55000 births a year. 
Table 1|Characteristics of mothers and births by deprivation category in Scotland 1994-2003 for 532016 births (529 317 live births and 2699 stillbirths). Values are percentages

\begin{tabular}{|c|c|c|c|c|c|}
\hline \multirow[b]{2}{*}{ Characteristic } & \multicolumn{5}{|c|}{ Deprivation category } \\
\hline & 1 (least) $(n=98745)$ & $2(n=96622)$ & $3(n=104260)$ & $4(n=110570)$ & 5 (most) $(n=121819)$ \\
\hline \multicolumn{6}{|l|}{ Maternal age: } \\
\hline$<20$ & 4.4 & 7.6 & 10.8 & 13.9 & 18.2 \\
\hline $20-24$ & 8.3 & 12.5 & 15.8 & 17.8 & 20.6 \\
\hline $25-29$ & 27.3 & 30.6 & 31.6 & 30.8 & 29.0 \\
\hline $30-34$ & 39.1 & 33.1 & 28.6 & 26.2 & 22.3 \\
\hline$\geq 35$ & 21.0 & 16.2 & 13.2 & 11.3 & 9.9 \\
\hline No previous births & 43.9 & 44.4 & 45.5 & 45.4 & 44.4 \\
\hline \multicolumn{6}{|l|}{ Obstetric intervention: } \\
\hline Spontaneous labour & 59.8 & 59.4 & 59.5 & 58.8 & 59.5 \\
\hline Induced labour & 24.6 & 25.7 & 26.1 & 26.6 & 26.6 \\
\hline Elective caesarean & 7.7 & 7.1 & 6.6 & 7.0 & 6.5 \\
\hline Emergency caesarean & 7.9 & 7.7 & 7.9 & 7.7 & 7.4 \\
\hline \multicolumn{6}{|l|}{ Smoking during pregnancy: } \\
\hline Yes & 13.2 & 19.6 & 24.6 & 29.7 & 38.4 \\
\hline No & 80.0 & 71.5 & 64.9 & 60.0 & 51.5 \\
\hline Not known & 6.8 & 8.9 & 10.5 & 10.3 & 10.2 \\
\hline Preterm & 4.8 & 5.3 & 5.7 & 6.1 & 6.9 \\
\hline Birth weight $<2500 \mathrm{~g}$ & 4.1 & 4.6 & 5.5 & 6.1 & 7.6 \\
\hline Male infants & 51.2 & 51.3 & 51.0 & 51.2 & 51.4 \\
\hline
\end{tabular}

We extracted records of all stillbirths and live singleton births delivered at 24-44 weeks' gestation in 1994-2003 ( $\mathrm{n}=541557)$. We chose this period because information on maternal smoking was not collected before 1994 . We then excluded babies with recorded birth weights of less than $500 \mathrm{~g}$ or more than $6499 \mathrm{~g}$, mothers recorded as 10 years old or younger, and records that were missing data on area deprivation score, infant sex, maternal age, or parity. We therefore included data on 529317 live births and 2699 stillbirths during 1994-2003. Information on deprivation score was missing in $1.7 \%$ of records. Smoking during pregnancy was coded as "no," "yes," or "not known." As $9.4 \%$ of the records for smoking were either missing or recorded as unknown and the characteristics of the "not known" group were of interest to us, we did not exclude them from the analysis but treated them as a distinct group. For all other variables, less than $0.1 \%$ of the data were missing.

Stillbirth was defined and applied after 1992 in Scotland as "a child which has issued forth from its mother after the twenty-fourth week of pregnancy and which did not at any time after being completely expelled from its mother breathe or show any other signs of life." ${ }^{17}$ Infant deaths were subdivided into death of a liveborn infant within the first 28 days of life (neonatal deaths) and death of a liveborn infant after the first 28 days of life but before 1 year of age (post-neonatal deaths).

Gestational age at birth was reported in completed weeks and based on the best estimate of gestation at birth from an ultrasound dating scan, the date of the last menstrual period, or both. Preterm delivery was defined as delivery at less than 37 weeks' completed gestation. Socioeconomic status was categorised by using quintiles of area based deprivation scores derived from postcode sector Carstairs-Morris scores for the whole population (using 2001 census data). ${ }^{18}$ Deprivation category 1 corresponds to the least deprived fifth, and category 5 corresponds to the most deprived fifth. We created a variable called "obstetric intervention" in a hierarchical way as follows. Any birth in which labour was induced was classed as an induction regardless of the final mode of delivery. Any delivery by caesarean section that was not induced we termed either emergency caesarean or elective caesarean. In other words, any references to deliveries after caesarean section were those that did not have induced labours. Low birth weight was defined as weighing $2500 \mathrm{~g}$ or less at birth. Maternal age at time of birth was grouped into five age bands, and parity was classified as "no previous births" or "one or more previous births."

We determined stillbirth rates as the number of stillbirths as a proportion of all live singleton births and stillbirths in the period and expressed the result per 1000 total births. We determined rates of infant, neonatal, and post-neonatal death as the number of deaths in each group as a proportion of all live singleton births in the period and expressed the result per 1000 live births. We determined the number of live births, stillbirths, and infant deaths in each deprivation category as well as the corresponding stillbirth and neonatal, post-neonatal, and infant death rates. We then calculated the odds ratios for stillbirth and for neonatal, post-neonatal, and infant death, comparing each deprivation category with the least deprived (reference) category. We used multiple logistic regression modelling to derive odds ratios adjusted for maternal age, parity, infant sex, and obstetric intervention (model A) and 
Table 2 | Numbers and rates of stillbirths and neonatal, post-neonatal, and infant death by deprivation category in Scotland 1994-2003 for 532016 births

\begin{tabular}{|c|c|c|c|c|c|}
\hline \multirow[b]{2}{*}{ Deprivation category } & \multirow[b]{2}{*}{ (No of births) } & \multicolumn{4}{|c|}{ Rate (No) of deaths } \\
\hline & & Stillbirths* & Neonatal† & Post-neonatal $\dagger$ & Infant $†$ \\
\hline 1 (least) & 98745 & $3.8(372)$ & $2.2(220)$ & $0.9(91)$ & $3.2(311)$ \\
\hline 2 & 96622 & $4.7(450)$ & $2.2(213)$ & $1.1(104)$ & $3.3(317)$ \\
\hline 3 & 104260 & $5.3(555)$ & $2.6(275)$ & $1.5(153)$ & $4.1(428)$ \\
\hline 4 & 110570 & $5.5(606)$ & $2.5(271)$ & $1.8(198)$ & $4.3(469)$ \\
\hline 5 (most) & 121819 & $5.9(716)$ & $3.1(371)$ & $2.5(286)$ & $5.4(657)$ \\
\hline All & 532016 & $5.1(2699)$ & $2.6(1350)$ & $1.6(832)$ & $4.1(2182)$ \\
\hline
\end{tabular}

for maternal age, parity, infant sex, obstetric intervention, and smoking status (model B).

The contribution of smoking in explaining the social inequalities in outcome between the most and the least deprived categories was calculated by using the method outlined by Singh-Manoux et al. ${ }^{19}$ Contribution of smoking $=100 \times\left(\mathrm{OR}_{\mathrm{B}}-\mathrm{OR}_{\mathrm{A}}\right) /\left(\mathrm{OR}_{\mathrm{A}}-1\right)$, where $\mathrm{OR}_{\mathrm{A}}$ is the odds ratio of outcome in deprivation category 5 compared with deprivation category 1 adjusted for year of birth, maternal age, parity, infant sex, and primary obstetric intervention and $\mathrm{OR}_{\mathrm{B}}$ is the odds ratio of outcome in deprivation category 5 compared with deprivation category 1 adjusted for the above variables plus smoking during pregnancy. This calculation generates a quantity that specifically identifies the relative contribution of smoking, as a risk factor, in explaining social inequalities in stillbirths and infant deaths. It differs from the measure known as population attributable risk, which estimates the reduction in stillbirths and deaths that would be seen if the entire population of prenatal women did not smoke. We used SAS, version 9.1 for all analyses.

\section{RESULTS}

The most deprived mothers tended to be younger and to be more likely to smoke and to give birth to preterm or low birthweight babies (table 1). Equally, the least deprived mothers were more likely to be older, nonsmokers, and less likely to give birth to preterm or low birthweight babies. Women in the most deprived category were three times more likely to smoke during pregnancy than were those in the least deprived category $(38 \% v 13 \%)$.

Overall, 2699 stillbirths and 2182 infant deaths occurred, corresponding to rates of 5.1 stillbirths per

Table 3 | Numbers and rates of stillbirths and neonatal, post-neonatal, and infant death by smoking category in Scotland 1994-2003 for 532016 births

\begin{tabular}{lccccc} 
& & \multicolumn{4}{c}{ Rate (No) of deaths } \\
\cline { 3 - 6 } Smoking group & (No of births) & Stillbirths* & Neonatal $†$ & Post-neonatal & Infant $†$ \\
No & 344502 & $3.8(1313)$ & $2.1(731)$ & $1.0(343)$ & $3.1(1074)$ \\
\hline Yes & 137303 & $6.7(914)$ & $3.0(414)$ & $2.8(380)$ & $5.8(794)$ \\
\hline Not known & 50211 & $9.4(472)$ & $4.1(205)$ & $2.2(109)$ & $6.1(314)$ \\
\hline All & 532016 & $5.1(2699)$ & $2.6(1350)$ & $1.6(832)$ & $4.1(2182)$ \\
\hline *Rate per 1000 total births. & & & & \\
†Rate per 1000 live births. & & & & \\
\hline
\end{tabular}

tRate per 1000 live births.
1000 total births and 4.1 infant deaths per 1000 live births. However, for stillbirths, the rate increased from 3.8 per 1000 in the least deprived group to 5.9 per 1000 in the most deprived group. We found a similar pattern in the neonatal, post-neonatal, and infant death rates (table 2).

The rates of stillbirth were lowest in the non-smokers and highest in the smoking "not known" category (table 3). We saw a similar pattern in the infant death rate and neonatal death rate.

The odds ratios for stillbirths and infant deaths in each deprivation category compared with the least deprived category clearly show a social gradient in all outcomes, with increasing odds related to increasing deprivation (table 4). Comparing mothers in the most deprived category with those in the least deprived category showed that stillbirths were $56 \%$ (odds ratio $1.56,95 \%$ confidence interval 1.38 to 1.77 ) more likely and that infant deaths were $72 \%(1.72,1.50$ to 1.97$)$ more likely in the most deprived group. Although an increased risk of neonatal death also existed in this group (37\%), the really striking finding is that post-neonatal deaths were two and half times more likely in the most deprived group (odds ratio 2.56, 2.02 to 3.24).

After adjustment for the differences in age distributions, parity, infant sex, and differences in obstetric intervention, the social gradient was slightly attenuated but still marked and statistically significant. After further adjustment for smoking during pregnancy, some attenuation in the gradient again occurred but a clear and significant trend was still apparent. Comparing mothers in the most deprived category with those in the least deprived category showed that stillbirths were $32 \%$ more likely and infant deaths were $35 \%$ more likely in the most deprived group. Assessment of the contribution of smoking to the inequality between the most and least deprived groups indicated that smoking accounted for about a third of the inequality: $38 \%$ of stillbirths and $31 \%$ of infant deaths.

\section{DISCUSSION}

We did a large population based retrospective cohort study of 532016 births in Scotland between 19942003, of which 2699 were stillbirths and 2182 resulted in an infant death. We found that the social inequalities in stillbirth and infant death in that time period were partly but not fully attenuated by adjustment for 
Table 4 | Odds ratios (95\% confidence intervals) of stillbirths and neonatal, post-neonatal, and infant deaths by deprivation category in Scotland 1994-2003, with contribution of smoking to the social inequality

$\begin{array}{llccc}\begin{array}{l}\text { Deprivation } \\ \text { category }\end{array} & \begin{array}{c}\text { Stillbirths } \\ (n=2699)\end{array} & \begin{array}{c}\text { Neonatal } \\ (n=1350)\end{array} & \begin{array}{c}\text { Post-neonatal } \\ (n=832)\end{array} & \begin{array}{c}\text { Infant } \\ (n=2182)\end{array}\end{array}$

1 (least) (reference

group):

\begin{tabular}{lllll}
\hline Unadjusted & 1 & 1 & 1 & 1 \\
\hline Model A* & 1 & 1 & 1 & 1 \\
\hline Model B十 & 1 & 1 & 1 & 1 \\
\hline
\end{tabular}

2:

\begin{tabular}{lllll} 
Unadjusted & $1.24(1.08$ to 1.42$)$ & $0.99(0.82$ to 1.20$)$ & $1.17(0.88$ to 1.55$)$ & 1.04 (0.89 to 1.22$)$ \\
\hline Model $A^{*}$ & $1.22(1.06$ to 1.40$)$ & $0.97(0.80$ to 1.17$)$ & $1.10(0.83$ to 1.45$)$ & $1.01(0.86$ to 1.18$)$ \\
\hline Model B $\dagger$ & $1.16(1.01$ to 1.33$)$ & $0.95(0.78$ to 1.14$)$ & $1.03(0.78$ to 1.37$)$ & $0.97(0.83$ to 1.13$)$ \\
\hline
\end{tabular}

3:

\begin{tabular}{|c|c|c|c|c|}
\hline Unadjusted & $1.42(1.24$ to 1.61$)$ & $1.19(0.99$ to 1.42$)$ & 1.60 (1.23 to 2.07$)$ & 1.31 (1.13 to 1.51$)$ \\
\hline Model $A^{*}$ & 1.39 (1.22 to 1.59$)$ & 1.14 (0.95 to 1.37$)$ & 1.42 (1.09 to 1.85$)$ & 1.22 (1.05 to 1.42$)$ \\
\hline Model B† & 1.27 (1.11 to 1.45$)$ & 1.09 (0.91 to 1.30$)$ & 1.28 (0.98 to 1.66$)$ & 1.14 (0.98 to 1.32$)$ \\
\hline \multicolumn{5}{|l|}{ 4: } \\
\hline Unadjusted & $1.46(1.28$ to 1.66$)$ & $1.10(0.92$ to 1.32$)$ & 1.95 (1.52 to 2.50$)$ & 1.35 (1.17 to 1.56$)$ \\
\hline Model $A^{*}$ & $1.42(1.25$ to 1.62$)$ & $1.05(0.87$ to 1.26$)$ & 1.65 (1.29 to 2.13$)$ & 1.23 (1.06 to 1.42$)$ \\
\hline Model B† & $1.28(1.12$ to 1.46$)$ & 0.99 (0.82 to 1.19$)$ & 1.44 (1.12 to 1.86$)$ & 1.13 (0.97 to 1.31$)$ \\
\hline \multicolumn{5}{|l|}{5 (most): } \\
\hline Unadjusted & $1.56(1.38$ to 1.77$)$ & 1.37 (1.16 to 1.62$)$ & 2.56 (2.02 to 3.24$)$ & 1.72 (1.50 to 1.97$)$ \\
\hline Model A* & 1.52 (1.34 to 1.73$)$ & $1.28(1.07$ to 1.51$)$ & 2.05 (1.61 to 2.61$)$ & $1.51(1.32$ to 1.74$)$ \\
\hline Model B† & 1.32 (1.16 to 1.50$)$ & 1.19 (1.00 to 1.41$)$ & 1.70 (1.33 to 2.17$)$ & 1.35 (1.17 to 1.55$)$ \\
\hline
\end{tabular}

Model $\mathrm{B} \dagger$

value):

\begin{tabular}{lcccc}
\hline Unadjusted & $<0.001$ & $<0.001$ & $<0.001$ & $<0.001$ \\
\hline Model A* $^{*}$ & $<0.001$ & 0.003 & $<0.001$ & $<0.001$ \\
\hline \multicolumn{1}{c}{ Model B† } & $<0.001$ & 0.04 & $<0.001$ & $<0.001$ \\
\hline $\begin{array}{l}\text { Contribution of } \\
\text { smoking }(\%) \S\end{array}$ & -38.46 & -32.14 & -33.33 & -31.17 \\
\hline
\end{tabular}

*Adjusted for year of birth, maternal age, parity, infant sex, primary obstetric intervention.

†Adjusted for year of birth, maternal age, parity, infant sex, primary obstetric intervention, smoking during pregnancy.

$\exists \mathrm{X}^{2}$ test of effect of fitting deprivation category as ordinal rather than categorical variable.

$\S$ Contribution of smoking to inequality between deprivation categories 5 and 1.

smoking during pregnancy and that smoking during pregnancy accounted for approximately a third of the inequality in stillbirths and infant deaths between the most and least deprived groups in the population. The gradient for neonatal deaths was less marked than those for stillbirths and post-neonatal deaths.

\section{Strengths and weaknesses}

The strength of our study is that it is population based and used one of the few national databases of routinely collected information. The coverage and quality of the data have been established to be good, and information on socioeconomic position and smoking is available. The Scottish morbidity record contains information on all births in Scottish hospitals. Given that less than $1 \%$ of births take place outside of hospital in Scotland, this database has excellent coverage; it is subject to regular quality assurance checks and is known to be $99 \%$ complete. ${ }^{20}$ We selected the period between January 1994 and December 2003, as the quality of the data are known to be very high from 1980 onwards, with very few errors and missing data during this time, and because the data on smoking have been collected only since 1994 .

Our study has some limitations that need to be considered. These concern the measurements of deprivation, smoking, and potential confounders. The use of area deprivation indices as measures of social inequality is well supported in the literature. ${ }^{21}$ These indices provide a complementary picture to individual measures of socioeconomic status and may be particularly useful in pregnant women, for whom determination of socioeconomic position is known to be problematic. ${ }^{22}$ However, an area based score does not always correspond to individually measured socioeconomic position, as some women who would be categorised in lower social classes live in affluent areas and many women who would be categorised in higher social classes live in deprived areas.

Previous research has indicated that women may stop, reduce, and sometimes restart smoking at various points during pregnancy. ${ }^{23}$ Given that nicotine dependence can make quitting smoking very difficult, attempts to maintain abstinence during pregnancy are unsurprisingly often unsuccessful. In addition, many women may be reluctant to disclose a perceived socially undesirable behaviour to their clinicians during pregnancy. As a result, recording the maternal report on smoking (yes/no) during pregnancy once at the initial booking visit will give only a snapshot (and quite possibly an unrepresentative one). Ideally, one would be able to study accurately quantified self reported smoking at various points before and during pregnancy along with salivary cotinine estimation. This has been achieved in several longitudinal studies, but collecting this information routinely for all women during pregnancy is simply not practicable. This must therefore be seen as a limitation of routinely collected data compared with smaller longitudinal studies. On the other hand, the outcomes of interest in this study (stillbirth and infant death) are comparatively rare, so few longitudinal studies are large enough to collect sufficient numbers to accurately and precisely estimate associations with these rare outcomes at the population level. We also consider that by including a non-responder category in this study we have to some extent tackled the problem of misclassification of smoking status. Our analyses show that women in this "not known" group have a similar risk profile to smokers. This suggests either that they are smokers who choose not to declare themselves as such or else that their "not known" status is a marker of a risk factor that has a similar magnitude to smoking. We consider the first of these to be more likely. Unfortunately, no studies from Scotland using cotinine validated self report against which to validate these figures have been published.

As most neonatal deaths are related to prematurity, the universal access to improvements in neonatal care seems to be reducing what might otherwise be a steeper social gradient for neonatal deaths. The apparent effects of smoking during pregnancy on post-neonatal 


\section{WHAT IS ALREADY KNOWN ON THIS TOPIC}

Rates of both stillbirth and infant death show social gradients within developed countries

Smoking during pregnancy has been clearly linked to stillbirth and infant deaths

Quantifying the contribution that smoking during pregnancy has on the social inequalities gap in stillbirths and infant deaths is of interest

\section{WHAT THIS STUDY ADDS}

Social gradients existed in the stillbirth and infant death rates in Scotland during 1994-2003

Smoking during pregnancy accounted for $38 \%$ of the inequality in stillbirths and $31 \%$ of the inequality in infant deaths

In addition to tackling smoking during pregnancy and reducing infants' exposure to tobacco smoke, other measures are needed to reduce social inequalities in these outcomes

deaths could be explained by a direct effect on the fetoplacental unit during pregnancy, influencing or programming neonatal health. However, smoking during pregnancy may also act as a "marker" for smoking in the postnatal period, which is an established risk for post-neonatal death, in particular for sudden infant death syndrome. ${ }^{24}$ As we did not have a measure of smoking in the postnatal period, we cannot assess the interplay of these factors.

Another limitation of the data was the lack of information on the use of alcohol and illicit drugs during pregnancy and on maternal pre-pregnancy weight. All these factors are related to both smoking and stillbirth/infant death. One could not argue that they are on the causal pathway between smoking and the outcomes, but they could be plausibly seen as potential confounders. On the other hand, although we had information on both birth weight and gestational age, we decided not to include either preterm birth or low birth weight in the models as we viewed them as potentially being on the causal pathways between smoking and death and our aim was to show the effect of smoking.

As far as we are aware, although several other studies have investigated the associations between measures of socioeconomic status, smoking during pregnancy, and adverse perinatal and infant outcomes, this is the first study using nationally collected data that has attempted to deconstruct and quantify the contribution of smoking to socioeconomic inequalities in both stillbirth and infant death. Similar analyses in countries with a lower prevalence of smoking would be very useful. The method we have used to quantify the contribution of smoking to social inequality is relatively straightforward to calculate and could be used in other studies. ${ }^{19}$

\section{Possible mechanisms and implications for clinicians and policymakers}

Despite recent policy interest in infant deaths, ${ }^{25}$ stillbirth accounts for a larger proportion of losses than do infant deaths, and in most cases the cause remains unclear. Reducing smoking during pregnancy may be one of the few modifiable risk factors for this outcome that might also reduce the social gradient. Both tackling smoking during pregnancy and reducing infants' exposure to tobacco smoke in the postnatal environment may help to reduce stillbirths and infant deaths overall, but because smoking is more prevalent in more deprived areas these measures may help to reduce the socioeconomic inequalities in stillbirths and infant deaths perhaps by as much as $30-40 \%$. Although the contribution of NHS smoking cessation services to reducing smoking prevalence has been small, it has had a disproportionate effect in the most disadvantaged areas, thus potentially reducing inequalities. ${ }^{26}$ Nevertheless, we would agree that more powerful and innovative targeted interventions are needed as well as action to strengthen tobacco control policy. ${ }^{27}$ Of course, many other considerations exist-for example, differential uptake of interventions and differential effects of antismoking policies such as smoking bans. If social gradients in the risk of stillbirths and infant death were partly attenuated by adjustment for smoking, this would be useful support for the use of antismoking measures to reduce the social gradients in these outcomes.

Finally, even after taking smoking into account, most of the effects of social deprivation are unexplained. Therefore, action on smoking, although necessary, is not in itself sufficient; other measures to improve the social circumstances, social support, and health of mothers and infants are needed..$^{28}$

We thank Edmund Hey for helpful comments on an earlier draft. Contributors: RG, SRB, JC, IG, SJ, and CW participated in the design, analysis, interpretation, and reporting of the findings. JJK participated in the analysis, interpretation, and reporting of the findings. All authors approved the final version. RG is the guarantor.

Funding: Partly funded by Chief Scientist Office project grant CZH/4/293. The funder had no part in the study design; in the collection, analysis, and interpretation of data; in the writing of the report; and in the decision to submit the paper for publication.

Competing interests: None declared.

Ethical approval: This was an analysis of routinely collected data for which Information Services Division (ISD) Scotland has overall permission. Permission for the necessary record linkage was granted by ISD's Privacy Advisory Committee.

1 Chalmers I. Short, Black, Baird, Himsworth, and social class differences in fetal and neonatal mortality rates. $B M J$ 1985;291:231-3.

2 Parsons L, Duley L, Alberman E. Socio-economic and ethnic factors in stillbirth and neonatal mortality in the NE Thames Regional Health Authority (NETRHA) 1983. Br J Obstet Gynaecol 1990;97:237-44.

3 Finch BK. Early origins of the gradient: the relationship between socioeconomic status and infant mortality in the United States. Demography 2003;40:675-99.

4 Olsen O, Madsen M. Effects of maternal education on infant mortality and stillbirths in Denmark. Scand J Public Health 1999;27:128-36.

5 Jørgensen T, Mortensen LH, Andersen AM. Social inequality in fetal and perinatal mortality in the Nordic countries. Scand J Public Health 2008;36:635-49.

6 Singh GK, Kogan MD. Persistent socioeconomic disparities in infant, neonatal, and postneonatal mortality rates in the United States, 1969-2001. Pediatrics 2007;119:e928-39.

7 Department of Health. Review of the health inequalities infant mortality PSA target. DH, 2007.

8 Salihu HM, Wilson RE. Epidemiology of prenatal smoking and perinatal outcomes. Early Hum Dev 2007;83:713-20.

9 Högberg L, Cnattingius S. The influence of maternal smoking habits on the risk of subsequent stillbirth: is there a causal relation? $\mathrm{Br}$ J Obstet Gynaecol 2007;114:699-704.

10 Fleming P, Blair PS. Sudden infant death syndrome and parental smoking. Early Hum Dev 2007;83:721-5.

11 Kleinman JC, Pierre MB, Madans JH. The effects of maternal smoking on fetal and infant mortality. Am J Epidemiol 1988;127:274-82. 
12 Schneider S, Schütz J. Who smokes during pregnancy? A systematic literature review of population-based surveys conducted in developed countries between 1997 and 2006. Eur J Contracept Reprod Health Care 2008;13:138-47.

13 Rush D, Cassano P. Relationship of cigarette smoking and social class to birth weight and perinatal mortality among all births in Britain, 5-11 April 1970. J Epidemiol Community Health 1983;37:249-55.

14 Stephansson O, Dickman PW, Johansson ALV, Cnattingius S. The influence of socio-economic status on stillbirth risk in Sweden. Int J Epidemiol 2001;30:1296-301.

15 Goy J, Dodds L, Rosenberg MW, King WD. Health-risk behaviours: examining social disparities in the occurrence of stillbirth. Paediatr Perinat Epidemiol 2008;22:314-20.

16 Gray R, Bonellie SR, Chalmers J, Greer I, Jarvis S, Williams C. Social inequalities in preterm birth in Scotland 1980-2003: findings from an area-based measure of deprivation. Br J Obstet Gynaecol 2008;115:82-90.

17 United Kingdom. Still-Birth Definition Act 1992 [16 March 1992]. Curr Law Statut Annot GB 1992;2:29-1-29-3.

18 Carstairs V, Morris R. Deprivation and health in Scotland. Aberdeen University Press, 1991.

19 Singh-Manoux A, Nabi H, Shipley M, Guéguen A, Sabia S, Dugravot A, et al. The role of conventional risk factors in explaining social inequalities in coronary heart disease: the relative and absolute approaches to risk. Epidemiology 2008;19:599-605.
20 Cole SK. Scottish maternity and neonatal records. In: Chalmers I, Mcllwaine GM, eds. Perinatal audit and surveillance. Royal College of Obstetricians and Gynaecologists, 1980:39-51.

21 Galobardes B, Lynch J, Davey Smith G. Measuring socioeconomic position in health research. Br Med Bull 2007;81-82:21-37.

22 Fairley L, Leyland AH. Social class inequalities in perinatal outcomes: Scotland 1980-2000. J Epidemiol Community Health 2006;60:31-6.

23 Pickett KE, Rathouz PJ, Kasza K, Wakschlag LS, Wright R. Selfreported smoking, cotinine levels, and patterns of smoking in pregnancy. Paediatr Perinat Epidemiol 2005;19:368-76.

24 Moon RY, Horne RS, Hauck FR. Sudden infant death syndrome. Lancet 2007;370:1578-87.

25 Department of Health. Implementation plan for reducing health inequalities in infant mortality: a good practice guide. DH, 2007.

26 Bauld L, Judge K, Platt S. Assessing the impact of smoking cessation services on reducing health inequalities in England: observational study. Tob Control 2007;16:400-4.

27 Steinberg MB, Schmelzer AC, Richardson DL, Foulds J. The case for treating tobacco dependence as a chronic disease. Ann Intern Med 2008;148:554-6.

28 Lu MC, Halfon N. Racial and ethnic disparities in birth outcomes: a life course perspective. Matern Child Health J 2003;7:13-30.

Accepted: 9 May 2009 